\title{
Nota Científica \\ Estimativa da resistência e rigidez da madeira de Eucalyptus grandis por meio de onda ultrassonora
}

\author{
Magnos Alan Vivian', Elio José Santini², Karina Soares Modes ${ }^{3}$, Douglas Edson Carvalho², Weslley Wilker Corrêa Morais ${ }^{4}$ \\ Joel Telles de Souza², Felipe Susin² \\ ${ }^{1}$ Escola Superior de Agricultura Luiz de Queiroz, Universidade de São Paulo (ESALQ/USP), Av. Pádua Dias, 11, CEP 13418-900, Piracicaba, SP, Brasil \\ ${ }^{2}$ Universidade Federal de Santa Maria (UFSM), Av. Roraima, 1000, CEP 97105-900, Santa Maria, RS, Brasil \\ ${ }^{3}$ Universidade Federal de Rondônia (UNIR), Campus Rolim de Moura, CEP 76940-000, Rolim de Moura, RO, Brasil \\ ${ }^{4}$ Universidade Estadual de Roraima (UERR), Campus São João da Baliza, Rua São Miguel, 177, CEP 69365-000, São João da Baliza, RR, Brasil
}

*Autor correspondente:

magnosalan@yahoo.com.br

Termos para indexação:

Ensaio não destrutivo

Módulo de elasticidade

Módulo de ruptura

Index terms:

Non-destructive testing

Modulus of elasticity

Modulus of rupture
Resumo - O presente estudo objetivou avaliar o uso da constante elástica dinâmica obtida no ensaio não destrutivo, como parâmetro para estimativa das propriedades mecânicas de rigidez e resistência da madeira de Eucalyptus grandis. Para isto, utilizou-se um emissor de ondas ultrassônicas, o qual teve sua sensibilidade avaliada através da comparação com os valores obtidos no ensaio destrutivo de flexão estática. Os resultados evidenciam que a constante elástica dinâmica pode ser utilizada para inferência não destrutiva da rigidez e resistência da madeira de Eucalyptus grandis, entretanto com ressalvas, pois os ajustes observados foram relativamente baixos, com $\mathrm{R}^{2}$ aj. de 0,25 e 0,39 , respectivamente.
Histórico do artigo:

Recebido em 28 abril 2011

Aprovado em 11 nov 2011

Publicado em 30 mar 2012

\section{Estimation of strength and stiffness of Eucalyptus grandis wood by ultrasound waves}

\begin{abstract}
This study aimed to evaluate the use of dynamic elastic constant obtained in non-destructive testing, as a parameter to estimate the mechanical properties of stiffness and resistance of Eucalyptus grandis. For this we used an emitter of ultrasonic waves, which had its sensitivity evaluated by comparison with the values obtained in the destructive test of static bending. The results show that the dynamic elastic constant can be used as non-destructive inference of the stiffness and resistance of Eucalyptus grandis, though with reservations, because the adjustments observed were relatively low, with $\mathrm{R}^{2}$ adj. 0.25 and 0.39 , respectively.
\end{abstract}

A caracterização das propriedades da madeira é feita por meio da avaliação destrutiva de corpos de prova, o que pode resultar na variabilidade dos resultados devido a fatores como uma amostragem inadequada ou problemas na preparação dos mesmos.

Novas tecnologias estão surgindo para avaliação das características da madeira, dentre as quais, segundo Ross (1999), os raios-X, a análise química, as propriedades vibracionais e transmissão de ondas sonoras, que podem ser usadas para avaliação não-destrutiva da madeira.
Os métodos não-destrutivos fornecem informações de forma eficaz e rápida, não sendo necessária a extração de corpos de prova, uma vez que a avaliação é feita na própria peça ou estrutura. Ross et al. (1998) definem o método não destrutivo como sendo uma forma de identificar as propriedades físicas e mecânicas de uma peça de determinado material sem alterar suas capacidades de uso final.

As avaliações destrutivas são, em geral, os métodos mais utilizados para a determinação das propriedades 
físicas e mecânicas da madeira. A obtenção dos resultados a partir dessas metodologias, por vezes, se torna inviável em razão do tempo consumido para a confecção dos corpos de prova e custo do material. Em contrapartida, as avaliações não destrutivas permitem a obtenção de informações precisas e amplas, medindo-se um maior número de amostras, em função da rapidez dos testes (Stangerlin et al., 2008).

A técnica de emissão de ondas de ultrassom tem sido testada, dentre outras finalidades, sobre o efeito e controle do teor de umidade da madeira (Costa, 2005; Calegari et al., 2007), avaliação da qualidade de peças de madeira (Miná et al., 2004; Carreira et al., 2006) e estimativa das propriedades mecânicas da madeira (Oliveira, 2001; Nogueira, 2003).

De acordo com Stangerlin et al. (2010), embasado nos princípios da propagação de ondas mecânicas, a avaliação não destrutiva de madeiras por meio da técnica de emissão de ondas de ultrassom, surge como sendo um dos métodos mais aplicados e promissores, em função da facilidade de operação e custo relativamente baixo na aquisição e operacionalização do equipamento, quando comparado aos equipamentos utilizados em ensaios destrutivos.

Bucur \& Böhnke (1994) citam que diversos fatores influenciam a propagação de ondas ultrassônicas na madeira, dentre os quais, as propriedades físicas do substrato, características anatômicas da espécie, condições do meio (temperatura e umidade) e procedimento utilizado para a tomada das medidas.

Conforme Erikson et al. (2000), a avaliação não destrutiva é uma importante ferramenta para a caracterização da madeira, podendo ser utilizada pelas indústrias para melhorar o controle de qualidade dos processos através de uma maior uniformidade na matéria-prima e em seus derivados.

O ultrassom é caracterizado pelo emprego de frequências acima de $20.000 \mathrm{~Hz}$. De acordo com Oliveira (2001), entre as vantagens de sua utilização, destacamse o baixo custo de aquisição do equipamento quando comparado ao das máquinas de classificação automática e o treinamento relativamente simples da mão de obra para utilização do equipamento, possibilitando que o método seja facilmente difundido em revendedores e indústrias de madeiras e derivados.

Segundo Wang et al. (2007), esforços significativos vêm sendo direcionados para o desenvolvimento de uma tecnologia consistente de avaliação não destrutiva capaz de predizer com eficácia as propriedades intrínsecas da madeira.

Com este intuito, o presente trabalho teve por objetivo avaliar o uso da constante elástica dinâmica obtida por meio de ensaio não destrutivo, como parâmetro na estimativa das propriedades mecânicas de rigidez e resistência da madeira de Eucalyptus grandis.

A madeira utilizada neste estudo foi obtida em um povoamento pertencente à Empresa Flosul Madeiras, localizada no Município de Capivari do Sul, RS, com clima "Cfa" de acordo com a classificação de Köppen. As árvores de Eucalyptus grandis, com 16 anos de idade, foram abatidas, transformadas em toras de $2 \mathrm{~m}$ de comprimento e transportadas para a serraria da empresa. $\mathrm{Na}$ serraria, as toras foram desdobradas em tábuas com as dimensões de 2,2 × 9,0 × 200,0 cm (espessura, largura e comprimento), de acordo com os padrões utilizados pela Flosul para a produção de decks de madeira.

Em seguida, essas tábuas foram encaminhadas ao laboratório de produtos florestais (LPF) da Universidade Federal de Santa Maria (UFSM) e, com auxílio de uma serra circular, confeccionados os corpos de prova com as dimensões de 2,0 × 2,0 x 30,0 cm (radial, tangencial e longitudinal). Selecionaram-se 15 corpos de prova. Os materiais que apresentaram defeitos, como inclinação acentuada da grã, presença de nós e/ou medula, foram descartados.

Antes da realização dos ensaios destrutivos e não destrutivos, os corpos de prova foram mantidos em câmara climatizada (temperatura de $20{ }^{\circ} \mathrm{C} \pm 2{ }^{\circ} \mathrm{C}$ e umidade de $65 \% \pm 5 \%$ ) até a estabilização do teor de umidade de equilíbrio próximo a $12 \%$, para determinação da massa específica aparente, com base no peso e volume dos corpos de prova.

Os ensaios não destrutivos foram realizados em um equipamento de ultrassom dotado de transdutores de pontos secos com frequência de aproximadamente $45 \mathrm{kHz}$, que mede diretamente o tempo de propagação das ondas, em microssegundos $(\mu \mathrm{s})$.

As leituras das velocidades de propagação das ondas ultrassônicas foram realizadas no centro das amostras, na direção longitudinal da madeira. A partir da razão de duas leituras do tempo de propagação das ondas e a distância do trecho percorrido (comprimento real do corpo de prova), calculou-se a velocidade de propagação das ondas. A constante elástica dinâmica (Ed) foi calculada por meio do produto entre a velocidade de propagação e a massa específica da madeira (Eq. 1). 


$$
E d=V^{2} \cdot M E
$$

Ed - Módulo de elasticidade dinâmico (MPa); V Velocidade de propagação da onda $\left(\mathrm{m} \mathrm{s}^{-1}\right)$; ME - Massa específica da madeira a $12 \%$ de umidade $\left(\mathrm{kg} \mathrm{m}^{-3}\right)$.

Após os ensaios não destrutivos, os corpos de prova foram submetidos ao ensaio de flexão estática, conforme a norma COPANT 30:1-006 (Comisión Panamericana de Normas Técnicas, 1972), para avaliar a acurácia e a sensibilidade do método ultrassonoro, com posterior obtenção dos módulos de elasticidade (rigidez) e de ruptura (resistência). Utilizou-se de uma máquina universal de ensaios mecânicos, com capacidade de 20 t de carga, dotada de um computador acoplado com software específico para aquisição dos dados.

Para avaliação estatística dos resultados, foi aplicada análise de regressão, em que a variável independente foi a constante elástica dinâmica (Ed), obtida em ensaio de ultrassom, e a variável dependente, a propriedade mecânica de elasticidade ou rigidez (MOE) e de ruptura ou resistência (MOR), obtida em ensaio convencional de flexão estática.
Os resultados obtidos nos ensaios de flexão estática (destrutivo) e ultrassom (não destrutivo) para a madeira de Eucalyptus grandis estão apresentados na Tabela 1.

Os dados apresentaram variabilidade aceitável, abaixo de $10 \%$, apenas para massa específica (ME) e velocidade de propagação da onda ultrassonora (V SOM). Já a constante elástica dinâmica (Ed), o módulo de elasticidade (MOE) e de ruptura (MOR) apresentaram variação superior a este limite, entretanto podem ser considerados satisfatórios devido a madeira ser um material heterogêneo, que naturalmente apresenta certa variabilidade.

A V SOM ficou dentro da média relatada por outros pesquisadores (Simpson, 1998; Gonçalez et al., 2001; Puccini, 2002; Ballarin \& Nogueira, 2005), os quais citam valores entre 4.000 e $6.000 \mathrm{~m} \mathrm{~s}^{-1}$.

Na Figura 1 estão apresentados os modelos estatísticos gerados para a estimativa dos módulos de elasticidade e de ruptura obtidos no ensaio de flexão estática em função da constante elástica dinâmica obtida pelo ensaio de ultrassom.

Tabela 1. Valores mínimos, máximos, médios e desvios padrão obtidos nos ensaios de flexão estática e de ultrassom para a madeira de Eucalyptus grandis.

\begin{tabular}{|c|c|c|c|c|c|}
\hline & \multirow{2}{*}{$\begin{array}{c}\mathrm{ME} \\
\left(\mathrm{g} \mathrm{cm}^{-3}\right)\end{array}$} & \multicolumn{2}{|c|}{ Ensaio ultrassonoro } & \multicolumn{2}{|c|}{ Ensaio flexão estática } \\
\hline & & V SOM $\left(\mathrm{m} \mathrm{s}^{-1}\right)$ & Ed (MPa) & MOE (MPa) & MOR (MPa) \\
\hline Mínimo & 0,45 & $4.080,00$ & $7.993,51$ & $8.066,67$ & 62,81 \\
\hline Máximo & 0,58 & $4.890,00$ & $13.610,82$ & $13.842,68$ & 103,31 \\
\hline Médio & 0,53 & $4.384,67$ & $10.173,14$ & $11.125,53$ & 87,13 \\
\hline $\mathbf{S}$ & 0,05 & 244,51 & $1.678,39$ & $1.455,03$ & 12,69 \\
\hline CV\% & 9,25 & 5,58 & 16,50 & 13,08 & 14,56 \\
\hline
\end{tabular}

ME - massa específica; V SOM - velocidade de propagação da onda ultrassonora; Ed - constante elástica dinâmica; MOE - módulo de elasticidade ou rigidez; MOR - módulo de ruptura ou resistência; $\mathrm{s}$ - desvio padrão; CV \% - coeficiente de variação.
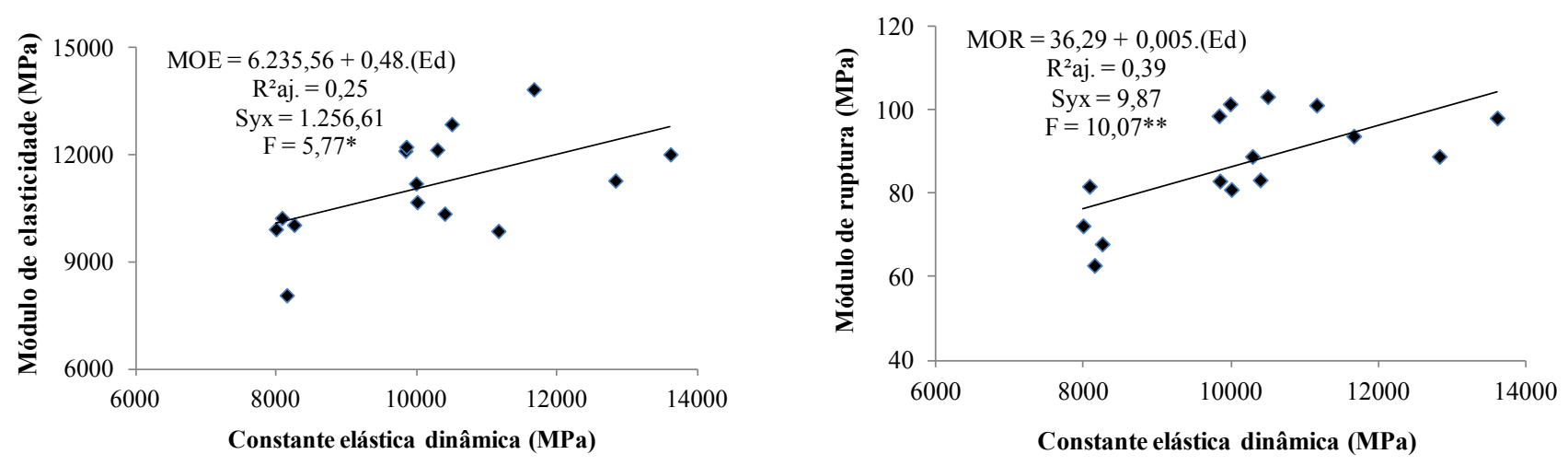

Figura 1. Modelo estatístico gerado para a estimativa do módulo de elasticidade ou rigidez e módulo de ruptura ou resistência a flexão estática em função da constante elástica dinâmica para a madeira de Eucalyptus grandis. 
Conforme Oliveira et al. (2005), existem correlações significativas para a estimativa do MOE em função do Ed, com coeficientes de determinação $\left(R^{2}\right)$ entre 0,57 e 0,89 . O valor do $R^{2}$ aj. encontrado no presente estudo para estimativa do MOE em função do Ed situa-se abaixo deste intervalo mencionado pelo autor, entretanto, foi significativo a $5 \%$ de probabilidade.

Com base nos parâmetros de regressão (maior coeficiente de determinação ajustado, menor erro padrão de estimativa e maior valor de F calculado), verificouse que o melhor ajuste foi na estimativa do módulo de ruptura. Acrescenta-se a isso que o ajuste foi significativo a $1 \%$ de probabilidade, enquanto o modelo gerado para estimativa do módulo de elasticidade apresentou significância de 5\%.

Gonçalez et al. (2001) também encontraram a mesma tendência, com modelos altamente significativos para a relação do módulo de ruptura com a constante elástica dinâmica, sendo superiores aos modelos gerados para o módulo de elasticidade. Bucur (1984) e Sandoz (1989) observaram o mesmo comportamento, com boas relações entre o módulo de ruptura e a constante elástica para outras espécies.

Já Halabe et al. (1995) e Stangerlin et al. (2010) encontraram resultado inverso. Ao correlacionarem os módulos de elasticidade e de ruptura obtidos nos ensaios mecânicos de diferentes espécies com as constantes elásticas dinâmicas estimadas mediante ensaios de propagação de ondas ultrassonoras, os ajustes foram superiores para o módulo de elasticidade em comparação com o módulo de ruptura. Segundo Oliveira et al. (2003) e Miná et al. (2004), esse comportamento está relacionado com a pequena tensão induzida na madeira durante os ensaios dinâmicos, ou seja, as medições dinâmicas são baseadas nas propriedades mecânicas apenas no limite elástico. Já para o módulo de ruptura, ocorre maior tensão depois do limite elástico, resultando, consequentemente, em predições menos eficazes por meio dos ensaios não destrutivos.

A correlação de Pearson estabelecida entre massa específica, flexão estática e ensaio ultrassonoro está apresentada na Tabela 2. Essa análise evidencia que as variáveis estão correlacionadas entre si, apresentando valores significativos, com exceção da V SOM que não se correlacionou com nenhuma das outras variáveis.
Tabela 2. Correlação de Pearson entre as variáveis na flexão estática e ensaio ultrassonoro para a madeira de Eucalyptus grandis.

\begin{tabular}{cccccc}
\hline & ME & MOE & MOR & V SOM & Ed \\
\hline ME & 1 & & & & \\
MOE & $0,74^{* *}$ & 1 & & & \\
MOR & $0,85^{* *}$ & $0,67^{* *}$ & 1 & & \\
V SOM & $0,25^{\text {ns }}$ & $0,22^{\text {ns }}$ & $0,30^{\text {ns }}$ & 1 & \\
Ed & $0,71^{* *}$ & $0,55^{*}$ & $0,66^{* *}$ & $0,86^{* *}$ & 1 \\
\hline
\end{tabular}

$\mathrm{ME}$ - massa específica, $\mathrm{g} \mathrm{cm}^{-3}$; MOE - módulo de elasticidade ou rigidez, MPa; MOR - módulo de ruptura ou resistência, MPa; V SOM - velocidade de propagação da onda ultrassonora, $\mathrm{m} \mathrm{s}^{-1} ; \mathrm{Ed}$ - constante elástica dinâmica, MPa. * - significativo ao nível de $5 \%$; ** - significativo ao nível de $1 \%$; ns - não significativo.

McDonald et al. (1990) encontraram correlação entre o módulo de elasticidade e a técnica de ondas acústicas. $\mathrm{O}$ autor afirma que é mais difícil relacionar módulo de ruptura com a constante elástica dinâmica, pois a presença de defeitos e a inclinação das fibras têm maior efeito que na velocidade longitudinal da onda ultrassonora. Entretanto, no presente trabalho, foi encontrada uma correlação superior do módulo de ruptura com a constante elástica dinâmica, quando comparada com o módulo de elasticidade.

Os resultados evidenciam que o uso da constante elástica dinâmica obtida na técnica ultrassônica pode ser utilizada para a inferência não destrutiva da rigidez e resistência da madeira de Eucalyptus grandis, entretanto, com ressalvas, pois os ajustes observados foram relativamente baixos, com $\mathrm{R}^{2} \mathrm{aj}$. de 0,25 e 0,39 , respectivamente.

\section{Referências}

BALLARIN, A. W.; NOGUEIRA, M. Determinação do módulo de elasticidade da madeira juvenil e adulta de Pinus taeda por ultra-som. Engenharia Agrícola, Jaboticabal, v. 25, n. 1, p. 19-28, 2005.

BUCUR, V. Ondes ultrasonores dans le bois: caractérisation mécanique et qualité de certaines essences de bois. 1984. $126 \mathrm{f}$. Thèse (Doctorat d'ingénieur) - ISMCM, St. Ouen, Paris.

BUCUR, V.; BÖHNKE, I. Factors affecting ultrasonic measurements in solid wood. Ultrasonics, Surrey, GB, v. 32, n. 5 , p. 385-390, 1994. 
CALEGARI, L.; STANGERLIN, D. M.; SANTINI, E. J; HASELEIN, C. R.; LONGHI, S. J.; CARMO, P. I. O.; SILVA FILHO, L. C. P.; GATTO, D. A. Monitoramento do teor de umidade de madeiras de Pinus elliottii Engelm. e Eucalyptus grandis W. Hill ex Maiden, sob diferentes temperaturas de secagem através do ultra-som. Ciência Florestal, Santa Maria, RS, v. 17, n. 4, p. 399-408, out./dez. 2007.

CARREIRA, M. R.; CHEUNG, A. B.; OLIVEIRA, F. G. R.; DIAS, A. A.; CALIL JÚNIOR, C.; SALES, A.; CANDIAN, M. Classificação de peças estruturais de pinus sp por ultra-som. In: CONGRESSO BRASILEIRO DE ENGENHARIA E CIÊNCIA DOS MATERIAIS, 17., 2006, Foz do Iguaçu. Resumos... [S.1.]: IPEN, 2006. 13 p.

COMISIÓN PANAMERICANA DE NORMAS TÉCNICAS. COPANT 30: 1-006: maderas: método de determinación de flexión estática. La Paz, 1972.

COSTA, O. A. L. Velocidade de propagação de ondas de ultrasom na madeira para diferentes condições de umidade. 2005. 92 f. Tese (Doutorado em Engenharia Agricola) - Universidade Estadual de Campinas, Campinas, SP.

ERIKSON, R. G.; GORMAN, T. M.; GREEN, D. W.; GRAHAM, D. Mechanical grading of lumber sawn from small-diameter lodgepole pine, ponderosa pine, and grand fir trees. Forest Products Journal, Madison, US, v. 50, n. 7/8, p. 59-65, 2000.

GONÇALEZ, J. C.; VALLE, A. T.; COSTA, A. F. Estimativas das constantes elásticas da madeira por meio de ondas ultra-sonoras (ultra-som). Cerne, Lavras, MG, v. 7, n. 2, p. 81-92, 2001.

HALABE, U. B.; BIDIGALU, G. M.; GANGARAO, H. V. S.; ROSS, R. J. Nondestructive evaluation of green wood using stress wave and transverse vibration techniques. Materials Evaluation, Evanston, US, v. 55, n. 9, p. 1013-1018, 1995.

MCDONALD, K. A.; FALK, R. H.; MALLORY, M. P. Nondestructive testing of wood products and structures: state of the art and research needs. Madison, WI: USDA, Forest Service, Forest Products Laboratory, 1990. p. 137-147.

MINÁ, A. J. S.; OLIVEIRA, F. G. R.; CALIL JUNIOR, C.; DIAS, A. A.; SALES, A. Avaliação não destrutiva de postes de madeira por meio de ultra-som. Scientia Forestalis, Piracicaba, SP, v. 1, n. 65, p. 188-189, 2004.

NOGUEIRA, M. Determinação de módulos de elasticidade à compressão da madeira de Pinus taeda L. com o uso de ultra-som. 2003. 165 f. Dissertação (Mestrado em Ciências Agronômicas) - Universidade Estadual Paulista Júlio de Mesquita Filho, Botucatu.
OLIVEIRA, F. G. R. Estudo de propriedades mecânicas de dicotiledôneas por meio de ensaios não destrutivos utilizando equipamento de ultra-som. 2001. 104 f. Dissertação (Mestrado em Ciência e Engenharia de Materiais) - Universidade de São Paulo, São Carlos, SP.

OLIVEIRA, F. G. R.; CANDIAN, M.; LUCCHETTE, F. F.; CALIL JUNIOR, C.; SALES, A. Avaliação de propriedades mecânicas de madeira por meio de ultra-som. In: PAN AMERICAN CONFERENCE FOR NONDESTRUCTIVE TESTING, 3., 2003, Rio de Janeiro. Resumos... Rio de Janeiro: [s.n.], 2003. 5 p.

OLIVEIRA, F. G. R. Influência da seção transversal na velocidade ultra-sônica na madeira de Eucalyptus citriodora. Cerne, Lavras, MG, v. 11, n. 2, p. 197-203, 2005.

PUCCINI, C. T. Avaliação de aspectos de qualidade da madeira utilizando o ultra-som. 2002. 139 f. Tese (Doutorado em Engenharia Agricola) - Universidade Estadual de Campinas, Campinas, SP.

ROSS, R. J.; BRASHAW, B. K.; PELLERIN, R. F. Nondestructive evaluation of wood. Forest Products Journal, Madison, US, v. 48, n. 1, p. 14-19, 1998.

ROSS, R. J. Using sound to evaluate standing timber. Forest Products Journal, Madison, US, Review, v. 49, p. 43-44, 1999.

SANDOZ, J. L. Grading of construction timber by ultrasound. Wood Science Technology, v. 23, n. 1, p. 95-108, 1989.

SIMPSON, W. T. Relationship between speed of sound and moisture content of red oak and hard maple during drying. Wood and Fiber Science, Madison, US, v. 30, n. 4, p. 405-413, 1998.

STANGERLIN, D. M.; CALEGARI, L.; SANTINI, E. J.; DOMINGUES, J. M. X.; GATTO, D. A.; MELO, R. R. Determinação do módulo de elasticidade em madeiras por meio de métodos destrutivo e não-destrutivo. Revista Brasileira de Ciências Agrárias, Recife, v. 3, n. 2, p. 145-150, 2008.

STANGERLIN, D. M.; GATTO, D. A.; MELO, R. R.; CALEGARI, L.; VIVIAN, M. A.; CASTELO, P. A. R; BELTRAME, R. Uso do ultrassom para estimativa das propriedades mecânicas da madeira de Peltophorum dubium. Ciência da Madeira, Pelotas, v. 1, n. 2, p. 44-53, 2010.

WANG, X.; CARTER, P.; ROSS, R. J.; BRASHA,W. Acoustic assessment of wood quality of raw forest materials: a path to increased profitability. Forest Products Journal, Madison, US, v. 57, n. 5, p. 6-14, 2007. 
